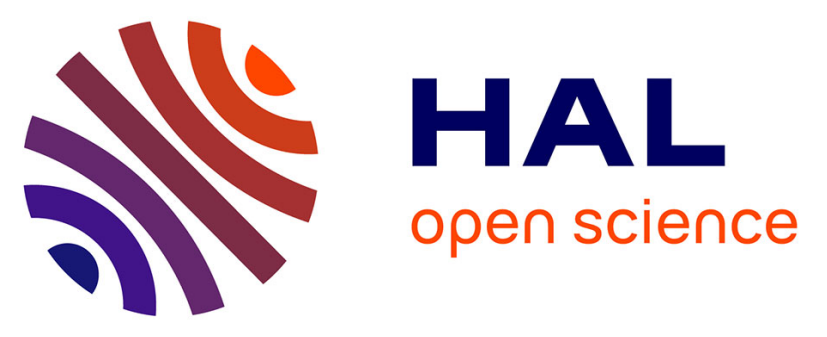

\title{
How Hydrogen and Oxygen Vapor Affect the Tribochemistry of Silicon- and Oxygen-Containing Hydrogenated Amorphous Carbon under Low-Friction Conditions: A Study Combining X-ray Absorption Spectromicroscopy and Data Science Methods
}

Filippo Mangolini, Komlavi Koshigan, Mark van Benthem, James Ohlhausen, John Mcclimon, James Hilbert, Julien Fontaine, Robert Carpick

\section{- To cite this version:}

Filippo Mangolini, Komlavi Koshigan, Mark van Benthem, James Ohlhausen, John Mcclimon, et al.. How Hydrogen and Oxygen Vapor Affect the Tribochemistry of Silicon- and Oxygen-Containing Hydrogenated Amorphous Carbon under Low-Friction Conditions: A Study Combining X-ray Absorption Spectromicroscopy and Data Science Methods. ACS Applied Materials \& Interfaces, 2021, 13 (10), pp.12610-12621. 10.1021/acsami.1c00090 . hal-03446611

\author{
HAL Id: hal-03446611 \\ https://hal.science/hal-03446611
}

Submitted on 24 Nov 2021

HAL is a multi-disciplinary open access archive for the deposit and dissemination of scientific research documents, whether they are published or not. The documents may come from teaching and research institutions in France or abroad, or from public or private research centers.
L'archive ouverte pluridisciplinaire $\mathbf{H A L}$, est destinée au dépôt et à la diffusion de documents scientifiques de niveau recherche, publiés ou non, émanant des établissements d'enseignement et de recherche français ou étrangers, des laboratoires publics ou privés. 


\title{
How Hydrogen and Oxygen Vapor Affect the Tribochemistry of Silicon- and Oxygen-Containing Hydrogenated Amorphous Carbon under Low
}

\author{
Friction Conditions: A Study Combining X-Ray Absorption \\ Spectromicroscopy and Data Science Methods
}

Filippo Mangolini ${ }^{1,2}$, Komlavi D. Koshigan ${ }^{3}$, Mark H. Van Benthem ${ }^{4}$, James A. Ohlhausen ${ }^{4}$, John B. McClimon ${ }^{5}$, James Hilbert ${ }^{6}$, Julien Fontaine ${ }^{3}$, Robert W. Carpick ${ }^{6, *}$

${ }^{1}$ Texas Materials Institute, The University of Texas at Austin, Austin, Texas 78712, USA

${ }^{2}$ Walker Department of Mechanical Engineering, The University of Texas at Austin, Austin, Texas 78712, USA

${ }^{3}$ Laboratoire de Tribologie et Dynamique des Systèmes, Ecole Centrale de Lyon, CNRS UMR 5513, Université de Lyon, 69134, Ecully cedex, France

${ }^{4}$ Sandia National Laboratories, Albuquerque, New Mexico 87185, USA

${ }^{5}$ Department of Materials Science and Engineering, University of Pennsylvania, Philadelphia, Pennsylvania 19104, USA

${ }^{6}$ Department of Mechanical Engineering and Applied Mechanics, University of Pennsylvania, Philadelphia, Pennsylvania 19104, USA

* Author to whom correspondence should be addressed. Electronic email: carpick@seas.upenn.edu 


\section{ABSTRACT}

The incorporation of silicon and oxygen into hydrogenated amorphous carbon $(\mathrm{a}-\mathrm{C}: \mathrm{H})$ is an effective approach to decrease the dependence of the tribological properties of a-C:H on the environment. Here, we evaluate the effect of hydrogen and oxygen partial pressure in vacuum on the tribological response of steel pins sliding against films consisting of silicon- and oxygencontaining a-C:H (a-C:H:Si:O). Experiments are conducted in the low friction/low wear regime, where sufficient gas pressure prevents steel from adhering to the a-C:H:Si:O, with the velocity accommodation mode being interfacial sliding between the tribotrack formed in the a-C:H:Si:O film and the carbonaceous tribofilm that is formed on the countersurface. The experiments indicated a decrease (increase) in friction and wear with the hydrogen (oxygen) pressure (hydrogen pressure between 50 and 2000 mbar; oxygen pressure between 10 and 1000 mbar). The X-ray photoelectron and absorption spectroscopic characterization of a-C:H:Si:O indicated tribologically-induced rehybridization of carbon-carbon bonds from $\mathrm{sp}^{3}$-to-sp $\mathrm{s}^{2}$. This mechanically-induced structural transformation coincided with the dissociative surface reaction between hydrogen (oxygen) gas molecules and $\mathrm{sp}^{2}$ carbon-carbon bonds that are highly-strained, which results in the formation of carbon-hydrogen groups (carbonyl or ether groups together with silicon atoms having higher oxidation states). On the basis of variations of the fraction of these surface functional groups with gas pressure, a phenomenological model is proposed for the gas pressure-dependence of friction for steel when sliding on a-C:H:Si:O films: while the decrease in friction with hydrogen pressure is induced by an increase in the percentage of carbon-hydrogen groups, the increase in friction with oxygen pressure is caused by a progressive increase in the relative fraction of silicon atoms having high oxidation states and surface oxygen concentration. 
KEYWORDS: Silicon- and oxygen-containing hydrogenated amorphous carbon; carbon-based materials; tribochemistry; solid lubrication; imaging near-edge X-ray absorption fine structure spectroscopy; NEXAFS; X-ray photoelectron spectroscopy; XPS. 


\section{INTRODUCTION}

Amorphous carbon (a-C)-based coatings have been used in several technological applications owing to their unique mechanical and physico-chemical properties coupled with the possibility of being deposited as smooth, conformal thin films ${ }^{1}$. The promising tribological properties (i.e., high wear resistance, low friction and adhesion) of a-C materials also made them candidate coatings for a range of applications, including as solid lubricants for aerospace components ${ }^{2}$, and as protective coatings for automotive parts ${ }^{3}$, high-performance tools ${ }^{4}$, electromechanical systems ${ }^{5}$, atomic force microscope probes ${ }^{6}$, and hard disk drives ${ }^{7}$.

Despite the attractive properties of a-C materials, their use in a range of application has been inhibited by two major limitations, namely the strong environmental dependence of their tribological properties $^{8-11}$, and their limited thermal stability ${ }^{1,12-13}$. In the case of a-C materials with significant amounts of hydrogen (usually referred to as hydrogenated amorphous carbon, a-C:H), low friction was typically observed in dry or vacuum conditions ${ }^{1-2}$, which was ascribed to the weak van der Waals interaction between a-C:H and the carbon-rich tribofilm (transfer layer) formed on metallic or ceramic countersurfaces ${ }^{14}$. However, friction increased upon sliding for prolonged periods of time: the depletion of hydrogen on the a-C:H surface induced by applied mechanical stresses was found to lead to $\mathrm{sp}^{3}$-to-sp ${ }^{2}$ rehybridization of carbon atoms present in the outermost layers $^{8-9,15}$. Upon exposure to molecular hydrogen, the excellent tribological properties of a-C:H were quickly recovered ${ }^{16}$. In contrast, the increase in friction of a-C:H in humid air was ascribed to the surface adsorption of water and/or oxygen ${ }^{10-11,17}$, leading to higher surface energies and to easier degradation of the surface.

In the case of hydrogen-free a-C, also called tetrahedral amorphous carbon (ta-C) when there is a sufficient degree of $\mathrm{sp}^{3}$-hybridized carbon present, the high friction and wear behavior observed 
in vacuum and/or dry environments ${ }^{1}$ was hypothesized to originate from the formation of covalent carbon-carbon bonds across the sliding interfaces following the mechanical removal of passivating surface species, such as hydrogen. The presence of gaseous species (e.g., hydrogen, oxygen, or water), which can dissociatively adsorb and saturate the dangling bonds formed during the sliding process, was shown to prevent reaching high friction and wear ${ }^{18}$, in agreement with molecular dynamics (MD) simulations ${ }^{19-20}$ and density functional theory calculations ${ }^{21-22}$ as well as with atomic force microscopy (AFM) studies ${ }^{23}$. Even though others proposed that ta-C materials exhibit low friction in humid environments by forming an ordered (graphitic) $\mathrm{sp}^{2}$-bonded carbon-rich surface layer ${ }^{24}$, Konicek et al. provided evidence supporting the passivation hypothesis using Xray photoemission electron microscopy (X-PEEM) with near-edge X-ray absorption fine structure (NEXAFS) spectroscopy ${ }^{25}$. Recently, Kunze et al. investigated by MD simulations and NEXAFS spectroscopy the wear process in ta-C- $v s .-$ ta-C tribosystems in dry conditions: both theory and experiments indicate that an amorphous carbon (a-C) layer with higher $\mathrm{sp}^{2}$ carbon content is formed ${ }^{26}$. The generation of this tribolayer in regions that have not experienced plastic yield, which was suggested as a precursor to wear events, clearly indicated the strong interplay of stress-induced rehybridization and repassivation by environmental species.

To improve the thermal stability and decrease the environmental dependence of the tribological behavior, dopants and alloying elements have been introduced in a-C materials ${ }^{13,27}$. Among the multiple doped and alloyed a-C materials synthesized so far, a-C:H materials that contain silicon and oxygen (i.e., silicon- and oxygen-containing hydrogenated amorphous carbon, a-C:H:Si:O) have attracted significant attention owing to their reduced residual stress $(<1 \mathrm{GPa})^{28}$, enhanced thermal stability ${ }^{29-30}$, and good tribological behavior in a wider range of environments compared to a-C: $\mathrm{H}^{28,31}$. The authors of the present study recently demonstrated that a-C:H:Si:O has higher 
thermal stability than a-C:H owing to carbon-silicon bonds, which have an equilibrium bond length that is longer compared to carbon-carbon $\mathrm{sp}^{3}$ bonds $^{32-33}$. This decreases the number of strained C$\mathrm{C}$ bonds present in a-C:H:Si:O, which could easily undergo bond scission at elevated temperatures. Under aerobic conditions, a-C:H:Si:O was also shown to exhibit a lower erosion rate than undoped a-C: $\mathrm{H}^{29-30}$ : the reaction between a-C:H:Si:O and oxygen or water molecules in the atmosphere results in the volatilization of carbon atoms present in the outermost surface region with the generation of a silica layer able to prevent further erosion of the underlying carbonaceous film ${ }^{34}$.

The promising tribological behavior of silicon-containing a-C:H (i.e., a-C:H:Si) and a-C:H:Si:O under different environmental conditions was evaluated by several research groups ${ }^{14,35-38}$. In the case of steel- $v s .-\mathrm{a}-\mathrm{C}: \mathrm{H}: \mathrm{Si}([\mathrm{Si}]=\sim 4$ at.\%), Gilmer and Hauert reported a much lower dependence of friction on environmental conditions ${ }^{38}$. Scharf et al. evaluated the tribological response of sapphire-vs.-a-C:H:Si:O sliding contacts by in situ Raman tribometry and ex situ time-of-flight secondary ion mass spectroscopy (ToF-SIMS) ${ }^{14,} 28,35-36$. The experimental results did not only indicate that the velocity accommodation mode (VAM) was interfacial sliding between the transfer layer (tribofilm) generated on the pin (either steel or silicon nitride countersurfaces) and a$\mathrm{C}: \mathrm{H}: \mathrm{Si}: \mathrm{O}$, but also provided evidence for a strong influence of the environment on the chemistry and shear strength of tribofilms formed on countersurfaces: in dry nitrogen, the interfacial layer was mostly made of carbon and had a lower shear strength ( $\sim 9 \mathrm{MPa})$, while under humid conditions it consisted of silica fragments and had a higher shear strength ( $78 \mathrm{MPa})$. These findings were proposed by the authors as the mechanism behind the higher coefficient of friction measured in humid air (around 0.2) compared to the one measured in dry nitrogen (0.092).

Recently, the authors of this work evaluated the tribological response of a steel pin sliding on a$\mathrm{C}: \mathrm{H}: \mathrm{Si}: \mathrm{O}$ under different environments ${ }^{37}$. While high friction was achieved at low hydrogen and 
oxygen gas pressures in vacuum $\left(\mathrm{H}_{2}\right.$ pressure $<50 \mathrm{mbar} ; \mathrm{O}_{2}$ pressure $\left.<10 \mathrm{mbar}\right)$, friction was low at higher gas pressures $\left(\mathrm{H}_{2}\right.$ pressure between 50 mbar and 1000 mbar; $\mathrm{O}_{2}$ pressure between 10 mbar and 500 mbar). The analysis of a-C:H:Si:O indicated that the tribolgical response of steelvs.-a-C:H:Si:O contacts is controlled by the formation of adhesive junctions at tribological interfaces: under vacuum conditions and low gas pressures, material is transferred from steel pins to a-C:H:Si:O, whereas at higher gas pressure a transfer film is formed on the steel countersurface. Imaging NEXAFS spectroscopy further indicated that upon sliding in oxygen (15 mbar) or hydrogen (100 mbar), i.e., under conditions for which low friction was measured, an $\mathrm{sp}^{2}$-bonded carbon-rich surface layer forms. These tribologically-induced changes in surface structure and chemistry indicated that hydrogen and oxygen dissociatively react with the tensile-strained $\mathrm{sp}^{2}$ carbon-rich layer at sliding interfaces and favor the release of adhesive junctions.

Even though the authors of the present study have already examined the role of hydrogen and oxygen in transitioning from a high- to a low-friction regime ${ }^{37}$, no systematic investigation has ever been reported about the phenomena taking place on a-C:H:Si:O when a steel pin slides on the film as a function of the partial pressure of oxygen and hydrogen gas. In particular, although sufficiently high gas pressures prevent material from steel pins to be transferred to a-C:H:Si:O and thus reduce friction substantially, the change in friction and wear in the low-friction regime as the gas pressures are further increased has not been explained. The present work aims to fill this gap by conducting tribological tests under different partial pressure of hydrogen and oxygen gas. The analysis of a-C:H:Si:O coatings by in situ (post mortem) XPS and ex situ NEXAFS spectromicroscopy, which also involved the use of advanced multivariate analysis methods, allowed the surface phenomena that occurred upon sliding to be investigated and correlated with the observed tribological response. 


\section{MATERIALS AND METHODS}

\subsection{Materials}

a-C:H:Si:O coatings (thickness: $2 \mu \mathrm{m}$ ) were grown on Si wafers by Sulzer-Metco Inc. (Amherst, NY, USA) by plasma-enhanced chemical vapor deposition (PECVD) process, which has already been described elsewhere ${ }^{28,31}$. The composition of the as-received films was $^{37,39}:[C]=57 \pm 3$ at. $\%$; $[\mathrm{H}]=34 \pm 3$ at. $\% ;[\mathrm{O}]=3 \pm 1$ at. $\% ;[\mathrm{Si}]=6 \pm 1$ at.\% (determined by Rutherford backscattering spectrometry together with hydrogen forward scattering spectroscopy, Evans Analytical Group, Sunnyvale, CA, USA). The fraction of $\mathrm{sp}^{2}$ carbon in the as-grown material was $0.48 \pm 0.02^{32}$ (determined by NEXAFS spectroscopy using the methodologies outlined in Ref. [40-41]). Additional information about the roughness, mechanical properties, density, and structure of a$\mathrm{C}: \mathrm{H}: \mathrm{Si}: \mathrm{O}$ can be found in Ref. [37, 39].

Consistent with previous work ${ }^{25}, 37,40,42-43$, reference carbon K-edge NEXAFS spectra were acquired on two materials with known fraction of $\mathrm{sp}^{2}$-bonded carbon, namely hydrogen-terminated ultrananocrystalline diamond (UNCD, Aqua 25, Advanced Diamond Technologies, Romeoville, IL, USA. Percentage of $\mathrm{sp}^{2}$-bonded carbon atoms: $7 \pm 3 \%{ }^{41}$ ) and highly ordered pyrolytic graphite (grade 2 HOPG, SPI Supplies, West Chester, PA, USA. Percentage of $\mathrm{sp}^{2}$-bonded carbon atoms: $100 \%)$

\subsection{Methods}

\subsubsection{Tribological Experiments under Controlled Environment}

Pin-on-flat linear reciprocating tests were carried out using a tribometer located in a high vacuum chamber that allows for gas introduction. Details about the tribological apparatus are reported in Ref. [37]. Steel (52100) pins with a curvature radius of $8 \mathrm{~mm}$ were employed in the present study. The root-mean square (RMS) roughness of the pins, measured by white light interferometry, was 
$27.3 \pm 0.2 \mathrm{~nm}$. Before the experiments, the pins were sonicated in acetone and isopropanol. Tribotests were carried out in high vacuum (without any baking) at room temperature for 500 passes. The applied normal load was $3 \mathrm{~N}$, which corresponds to a maximum Hertzian contact stress of $430 \mathrm{MPa}$ and an Hertzian contact radius of $60 \mu \mathrm{m}$. The stroke length and sliding speed were 2 $\mathrm{mm}$ and $2 \mathrm{~mm} \mathrm{~s}^{-1}$, respectively. All friction tests were started after achieving the desired gas pressure in the tribometer chamber. These tribological conditions were chosen to be consistent with relatively severe contact conditions found in moving mechanical components in applications found across several industrial sectors, including aerospace and automotive systems ${ }^{44-45}$.

To compute the friction coefficient, only data points (friction force and normal load) acquired while the pin position was between $1 / 8$ and $7 / 8$ of the stroke length were considered to avoid endpoint effects (see Figure S1 in the Supporting Information). The friction coefficient was computed as the ratio between the tangential force and the normal load. In this work, friction coefficient data acquired during one sliding cycle are averaged and reported together with the corresponding standard deviation.

\subsubsection{Near Edge X-Ray Absorption Fine Structure (NEXAFS) Spectromicroscopy}

Near edge X-ray absorption fine structure (NEXAFS) spectromicroscopic analyses were carried out at beamline U7A at the National Synchrotron Light Source (NSLS, Brookhaven National Laboratory, Upton, NY, USA) using the parallel processing imaging system available at the NIST/Dow endstation, which was extensively described in Ref. [46-47]. In brief, this analytical technique enables the characterization of the surface chemistry and elements' local bonding configuration with a $50 \mu \mathrm{m}$ lateral resolution and a $18 \times 13 \mathrm{~mm}^{2}$ field of view. 
The photon energy range and flux of beamline U7A is $100-800 \mathrm{eV}$ and $2 \times 10^{11}$ photons/second $/ 0.1 \%$ bandwidth, respectively. Data acquisition can only be performed in partial electron yield (PEY) mode. NEXAFS images were acquired at photon energy between $260 \mathrm{eV}$ and $340 \mathrm{eV}$ (carbon K-edge) with a $0.1 \mathrm{eV}$ energy resolution. The detector entrance grid bias (EGB) was varied between $50 \mathrm{~V}$ and $225 \mathrm{~V}$. Varying the EGB voltage allows the surface sensitivity of the NEXAFS measurements to be systematically changed ${ }^{41,48}$ : increasing the EGB voltage progressively filters out inelastically-scattered electron that have suffered energy loss while travelling through the sample, thus decreasing the escape depth of the collected electrons and the information depth of the measurements.

The calibration of the photon energy was performed using the $\mathrm{C} 1 \mathrm{~s} \rightarrow \pi^{*}$ signal of freshly-cleaved HOPG, which was set at $285.5 \mathrm{eV}$. All NEXAFS imaging data were processed using LDF software (Synchrotron Research Inc., Melbourne Beach, FL, USA). The NEXAFS data were normalized to the drain current simultaneously measured using a gold mesh located upstream from the analysis chamber. The images were then normalized to the pre-edge region intensity (intensity between 270 and $280 \mathrm{eV}$ ). The data were then normalized using the post-edge intensity (at $320 \mathrm{eV}$ ) so that any variation in spectral intensity is due to changes in chemical environment and independent on the number density of absorbing atoms.

\subsubsection{X-Ray Photoelectron Spectroscopy (XPS)}

The surface chemistry of a-C:H:Si:O was investigated using a Versa-Probe II (ULVAC-PHI, Chanhassen, MN, USA) X-ray photoelectron spectrometer directly attached to the tribometer chamber. The spectrometer was calibrated following the ISO 15472:2001 norm. The accuracy of the system was $\pm 0.1 \mathrm{eV}$. After tribological testing, the a-C:H:Si:O samples were transferred from 
the tribometer chamber to the XPS chamber in vacuum, i.e., without any exposure to air. In the present study, the analysis points were identified using a combination of optical microscopic images and X-ray induced secondary electron images (SXI). Small-area XPS spectra were collected in the constant-analyzer-energy (CAE) mode from both wear tracks and non-contact regions using a beam size of $18 \mu \mathrm{m}$ and a power of $4.3 \mathrm{~W}$. High-resolution XPS spectra were acquired with a pass energy of $23.5 \mathrm{eV}$ and step size of $0.1 \mathrm{eV}$. This acquisition settings resulted in a full-width-at-half-maximum of the $\mathrm{Ag} 3 \mathrm{~d}_{5 / 2}$ peak of $0.61 \mathrm{eV}$. Survey spectra were collected with a pass energy of $187.9 \mathrm{eV}$ and step size of $0.8 \mathrm{eV}$. The spectra were processed using CasaXPS software (version 2.3.16, Casa Software Ltd., Wilmslow, Cheshire, U.K.). An iterated ShirleySherwood background was subtracted before peak fitting. No sample charging was observed.

Quantitative analysis on the basis of XPS data was carried out using the method outlined in Ref. [49]. Briefly, the quantification of the surface composition was based on the integrated intensity (i.e., the peak area in Cps $\mathrm{x} \mathrm{eV}$ ) using a first principles model using Powell's equations ${ }^{50}$. The inelastic mean free path (IMFP) was computed using the TPP-2M formula ${ }^{51}$.

All XPS results reported herein are mean values computed from three measurements. The corresponding standard deviation is also reported.

\subsubsection{Multivariate Analysis of Imaging NEXAFS Data}

NEXAFS spectroscopic data were exported from LDF software as ASCII files and processed using Sandia National Laboratories authored programs written in Matlab ${ }^{\circledR}$ (MathWorks ${ }^{\circledR}$, Natick, MA). Data pretreatment included cropping images to include the center-of-field sample surface where tribological experiments were performed. After spatial median filtering to remove outliers, the raw data were first normalized to the current measured using a gold mesh upstream from the 
analysis chamber. A pre-/post-edge normalization was then performed. A detailed description of methodologies for the subsequent multivariate analyses (MVA) is reported in Ref. [52-53]. Briefly, prior to principal component analysis (PCA) ${ }^{54-55}$, the data were Poisson-scaled in spatial and spectral domains using the inverse of the square-roots of their respective means ${ }^{56}$. Following scaling, the data were subjected to eigenanalysis to evaluate the pseudorank. After estimating a rank for decomposition, data reduction and factor analysis was performed using PCA. The factors were then unscaled, reorthogonalized, and rotated using varimax rotation ${ }^{53,55}$ to impose spatial simplicity. In addition to PCA and varimax rotation, factors were estimated using multivariate curve resolution (MCR-ALS) $)^{57}$ and PARAFAC ${ }^{58}$.

PCA can be represented in matrix form as:

$D=T P^{T}+E$

Eq. 1

where $\boldsymbol{D}$ is the unfolded NEXAFS hyperspectral image data having dimension of $m \times n$. The rows of $\boldsymbol{D}$ are the image pixels, or spatial domain, and the columns are the energy channels, or spectral domain. For the case of a rank- $p$ decomposition, $\boldsymbol{T}$ is a $m x p$ matrix containing the orthogonal spatial factors of $\boldsymbol{D}$, and $\boldsymbol{P}$ is a $n \times p$ orthonormal matrix describing the spectral domain of $\boldsymbol{D}$. $\boldsymbol{E}$ is a $m x n$ matrix of residuals or noise, and the superscript $T$ indicates the transpose of the preceding matrix.

MCR can be represented in matrix form as:

$D=A S^{T}+E$

Eq. 2 
where $\boldsymbol{A}$ is a $m \times p$ matrix and $\boldsymbol{S}$ is a $n \times p$ matrix containing physically interpretable spatial and spectral domain factors, respectively, of $\boldsymbol{D}$. These factors are typically obtained by applying constraints such as non-negativity during an iterative alternating least squares process.

\section{RESULTS}

The average friction coefficient computed using the force values at steady-state (i.e., during the last 150 cycles $)$ is displayed as a function of gas $\left(\mathrm{O}_{2}\right.$ or $\left.\mathrm{H}_{2}\right)$ partial pressure in a log-log format in Figure 1a. While the coefficient of friction increased with the oxygen pressure (by a factor of 2.6 over 2 decades of pressure), it decreased upon increasing the hydrogen pressure (by a factor of 3.4 over 2 decades of pressure). Similarly to the friction results, the specific wear rates were strongly affected by the oxygen and hydrogen partial pressure: an increase in the partial pressure of oxygen (hydrogen) led to an increase (decrease) in the specific wear rate (Figure 1b) (by a factor of 5.1 (10.1) over 2 decades of pressure). To further evaluate the major role of the gaseous environment in affecting the tribological response of steel pins sliding against a-C:H:Si:O, the specific wear energy was calculated as the ratio between the cumulative dissipated energy (integral under the friction force vs. sliding distance curve) and the worn volume. While the specific wear energy significantly increased with the hydrogen pressure, it slightly decreased with the oxygen pressure (Figure 1c), by factors of 3 and 0.5 respectively over 2 decades of pressure. These findings clearly show that the tribological response of steel sliding against a-C:H:Si:O is critically dependent on environmental conditions, or, in other words, on gas pressure-dependent chemical process(es) and structural transformation(s) occurring at the steel/a-C:H:Si:O buried sliding interface. 

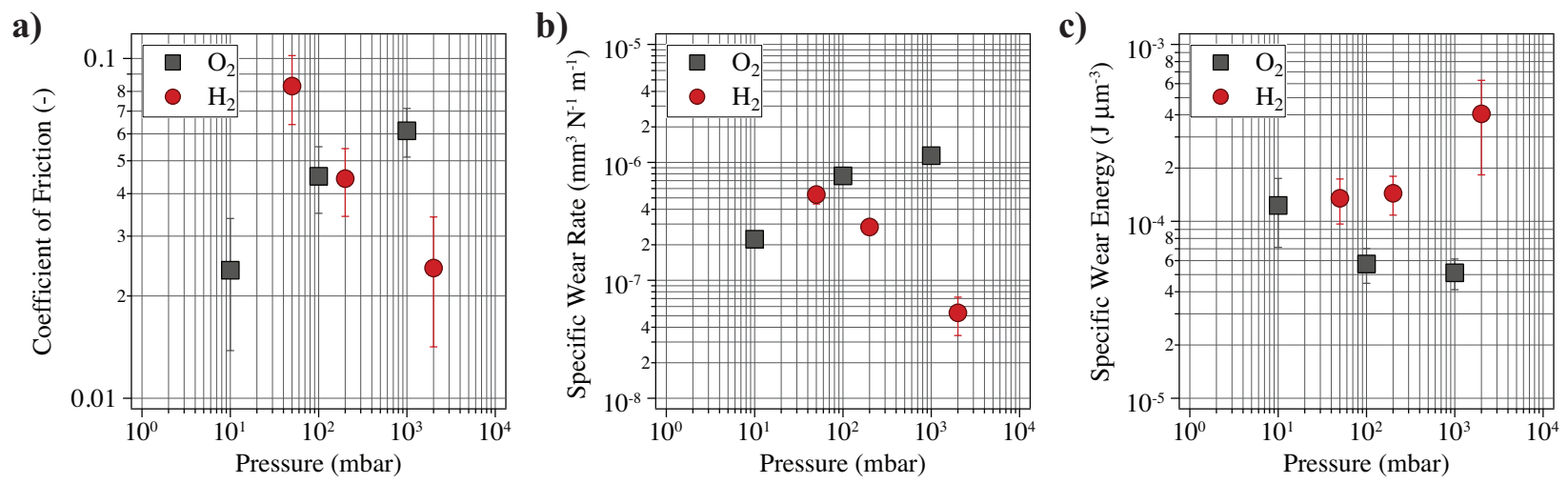

Figure 1. Steady-state coefficient of friction (a), specific wear rate (b), and specific wear energy (c) as a function of the partial pressure of hydrogen and oxygen gas.

To evaluate the surface phenomena occurring in a-C:H:Si:O during the sliding process under different environments, imaging NEXAFS analyses were carried out on a-C:H:Si:O films. Figure 2 displays representative NEXAFS chemical maps acquired at photon energies that correspond to characteristic NEXAFS absorption peaks of a-C:H:Si: $\mathrm{O}^{37}$. These photon energies were selected upon analyzing the typical spectrum of a-C:H:Si:O extracted from the non-contact region (Figure 3a) together with its second derivative spectrum (to easily identify characteristic spectral features). The NEXAFS maps suggested an increase in the fraction of $\mathrm{sp}^{2} \mathrm{C}-\mathrm{C}$ (disordered) bonds and $\mathrm{C}-\mathrm{H}$ functional groups inside the wear tracks independent of the environment in which the sliding experiments were carried out, as indicated by the increase in electron yield intensity in the NEXAFS maps acquired at 285.1 $\pm 0.2 \mathrm{eV}\left(\mathrm{C} 1 \mathrm{~s} \rightarrow \pi^{*}\right.$ transition for disordered C-C sp ${ }^{2}$ bonds $\left.{ }^{59-60}\right)$ and $287.6 \pm 0.2 \mathrm{eV}\left(\mathrm{C} 1 \mathrm{~s} \rightarrow \sigma^{*}\right.$ transition for $\mathrm{C}-\mathrm{H}$ groups $\left.{ }^{41-42,59}\right)$. A significant increase in the fraction of $\mathrm{C}=\mathrm{O}$ bonds was observed only in the case where the wear tracks were generated while sliding in oxygen, as indicated by the contrast in the NEXAFS map acquired at $286.7 \pm 0.2 \mathrm{eV}$, a photon 
energy corresponding to the $\mathrm{C} 1 \mathrm{~s} \rightarrow \pi^{*}$ feature of $\mathrm{C}=\mathrm{O}$ bonds containing a minor contribution from C-O Rydberg orbitals ${ }^{59,61}$.

a) $\mathrm{C} 1 \mathrm{~s} \rightarrow \pi^{*} \quad\left(\right.$ disordered $\left.\mathrm{sp}^{2} \mathrm{C}-\mathrm{C}\right)$

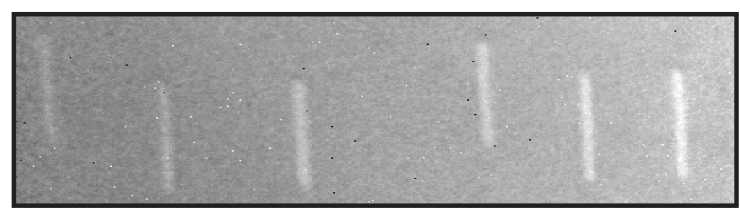

b) $\mathrm{C} 1 \mathrm{~s} \rightarrow \pi^{*} \quad(\mathrm{C}=\mathrm{O})$ and $\mathrm{C}-\mathrm{O}$ Ryd. orb.

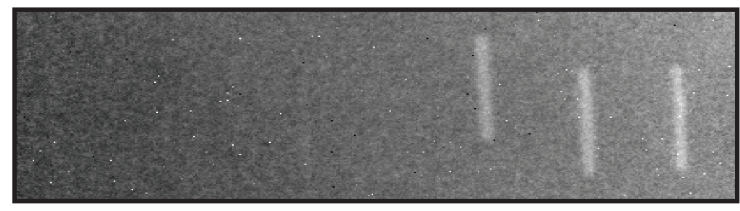

c) $\mathrm{C} 1 \mathrm{~s} \rightarrow \sigma^{*}(\mathrm{C}-\mathrm{H})$

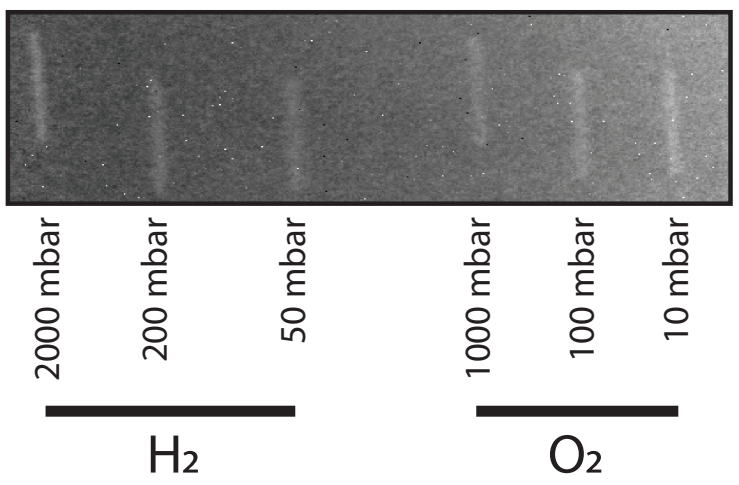

high

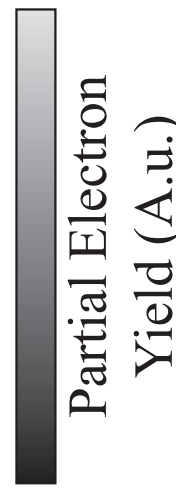

low

$1 \mathrm{~mm}$

Figure 2. NEXAFS chemical maps collected on an a-C:H:Si:O specimen on which six tribotests were carried out at different hydrogen and oxygen pressure. The maps were acquired at: (a) $285.1 \pm 0.2 \mathrm{eV}-\mathrm{C} 1 \mathrm{~s} \rightarrow \pi^{*}$ transition for disordered C-C sp ${ }^{2}$ bonds $^{59-60}$, (b) $286.7 \pm 0.2 \mathrm{eV}-\mathrm{C} 1 \mathrm{~s} \rightarrow \pi^{*}$ feature of $\mathrm{C}=\mathrm{O}$ bonds with a minor contribution from $\mathrm{C}-\mathrm{O}$ Rydberg orbitals ${ }^{59,61}$, (c) $287.6 \pm 0.2 \mathrm{eV}$ $-\mathrm{C} 1 \mathrm{~s} \rightarrow \sigma^{*}$ transition for $\mathrm{C}-\mathrm{H}$ groups ${ }^{41-42,59}$. 
The comparison of the NEXAFS data extracted from the contact region with the data obtained from the non-contact area indicated slight changes in electron yield for the characteristic absorption signals of a-C:H:Si:O upon sliding (Figure $3 b$ and Figure 3c, top part). Difference spectra (worn spectrum minus unworn spectrum) were assessed to easily identify sliding-induced variations in surface structure and chemistry of a-C:H:Si:O. The calculation of difference spectra enables the contribution of the adventitious contamination layer to the spectral intensity to be removed (assuming this layer on the specimen has a composition and thickens that is laterally homogeneous).

In the case of the tests carried out in hydrogen (Figure 3b), the intensity of the characteristic feature at $285.1 \pm 0.2 \mathrm{eV}\left(\mathrm{C} 1 \mathrm{~s} \rightarrow \pi^{*}\right.$ transition for disordered $\mathrm{C}-\mathrm{C} \mathrm{sp}{ }^{2}$ bonds $\left.{ }^{59-60}\right)$ increased in the wear tracks generated at 50 and 200 mbar, which indicates the conversion of $\mathrm{C}-\mathrm{C}$ bonds from $\mathrm{sp}^{3}$ to $\mathrm{sp}^{2}$, while decreased at 2000 mbar. Furthermore, the peak at $288.8 \pm 0.2 \mathrm{eV}$, which corresponds to the $\mathrm{C} 1 \mathrm{~s} \rightarrow \sigma^{*}$ transition for $\mathrm{C}-\mathrm{Si}$ and $\mathrm{C}-\mathrm{O}$ bonds, and to the $\mathrm{C} 1 \mathrm{~s} \rightarrow \pi^{*}$ transition for $\mathrm{C}=\mathrm{O}$ groups ${ }^{59}$, ${ }^{61-62}$, decreased in intensity. Since as-deposited a-C:H:Si:O does not contain C-O bonds ${ }^{32-33}$, the contribution of the contamination layer (containing $\mathrm{C}-\mathrm{O}$ bonds) is eliminated upon computing difference spectra and, thus, the signal appearing in difference spectra can be assigned to C-Si bonds. The computed difference spectra also exhibited intense positive signals at $286.0 \pm 0.2 \mathrm{eV}$ and $287.8 \pm 0.2 \mathrm{eV}$, which indicate the presence of a higher fraction of $\mathrm{C}=\mathrm{O}$ and $\mathrm{C}-\mathrm{H}$ functional groups in the wear track. The detection of a positive signal assigned to carbon-oxygen groups inside the wear tracks generated in hydrogen is due to residual oxygen and/or water in the vacuum chamber. However, the relative intensity of $\mathrm{C}-\mathrm{H}$ and $\mathrm{C}=\mathrm{O}$ signals increased with hydrogen pressure, which indicates a progressive surface enrichment of $\mathrm{C}-\mathrm{H}$ functional groups upon increasing the partial pressure of hydrogen while performing sliding tests against steel pins. 
As for the tests carried out in oxygen (Figure 3c), the fraction of $\mathrm{sp}^{2} \mathrm{C}-\mathrm{C}$ bonds increased in the wear tracks, as indicated by the positive peaks detected in carbon K-edge difference spectra. The negative peak at $288.8 \pm 0.2 \mathrm{eV}$ and the positive peak at $286.0 \pm 0.2 \mathrm{eV}$ respectively indicated a decrease in the number of C-Si bonds and an increase in the number of carbonyl/ether groups in the outermost region of a-C:H:Si:O while sliding in oxygen. Due to residual hydrogen (and/or water) in the vacuum chamber during the experiments performed in oxygen, the difference spectra also exhibited a positive signal for the characteristic feature of $\mathrm{C}-\mathrm{H}$ bonds at $287.8 \pm 0.2 \mathrm{eV}$. However, while no significant variations in the relative intensity of the peaks assigned to carbonyl/ether groups and carbon-hydrogen bonds was observed, a decrease in their absolute intensity occurred with gas pressure (note: the specific wear rate increased with gas pressure). 
a)

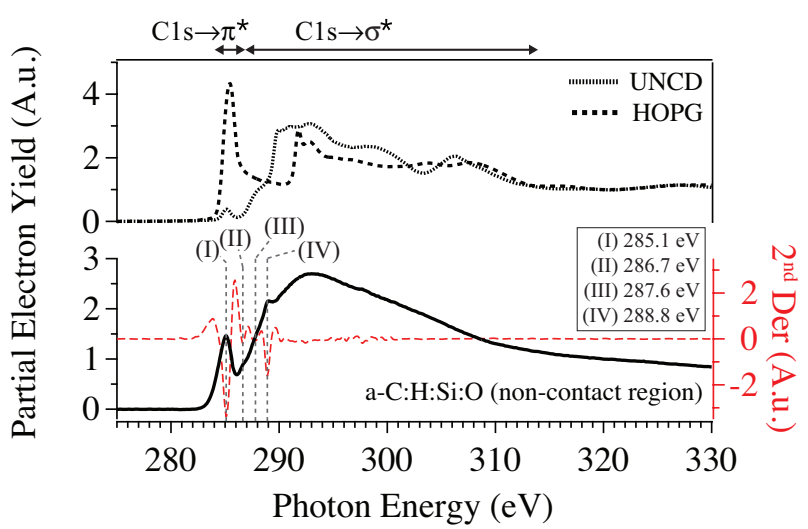

b)
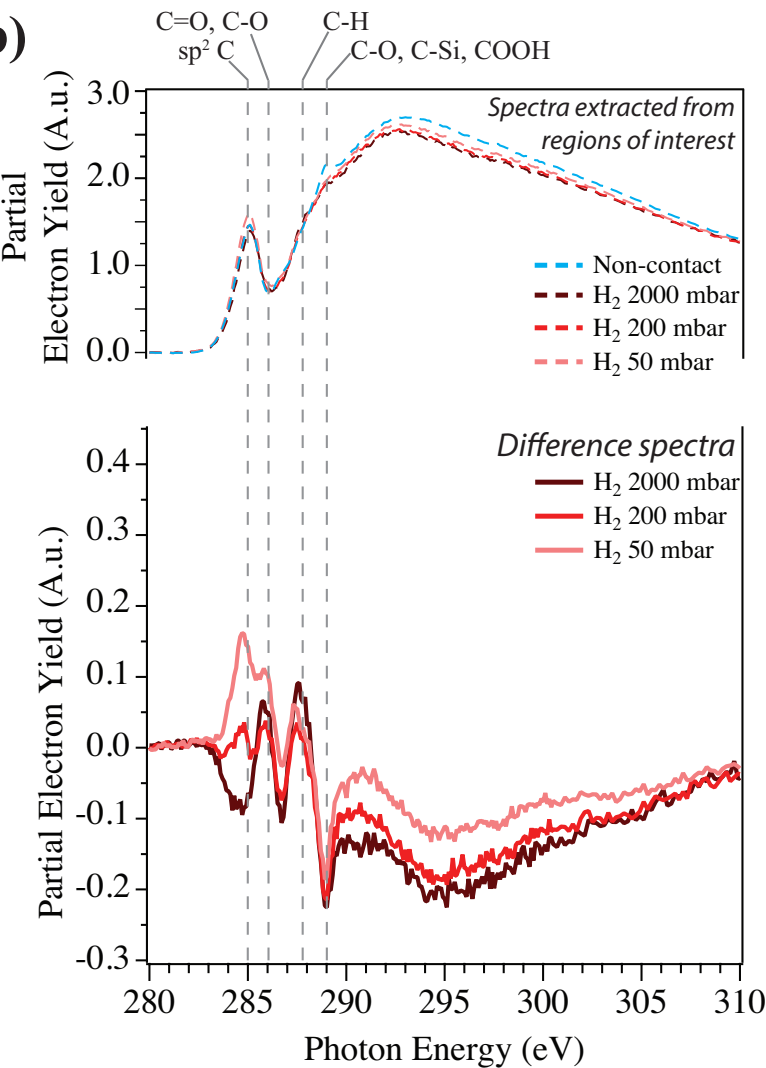

c)

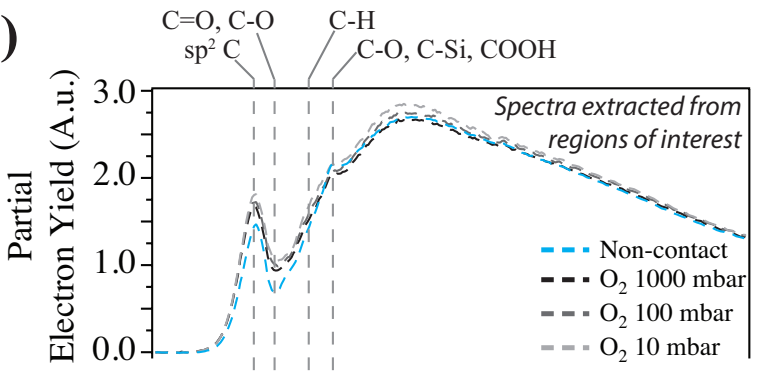

Difference spectra

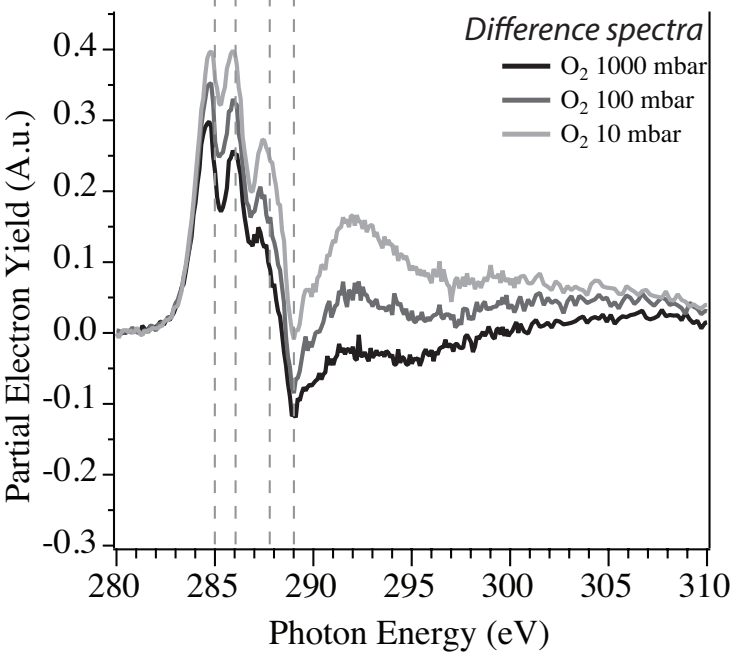

Figure 3. (a) Carbon K-edge NEXAFS spectrum of a-C:H:Si:O extracted from the non-contact region of the NEXAFS images reported in Figure 2. To identify spectral features (absorption bands and shoulders), the second derivative of the spectrum was computed (solid red line). The reference carbon K-edge spectra of UNCD and HOPG are also shown (dashed black lines); (b)-(c) carbon K-edge NEXAFS spectra extracted from non-contact and contact regions of the NEXAFS maps 
reported in Figure 2 together with difference spectra (difference in the carbon K-edge NEXAFS spectra extracted from worn and unworn regions of a-C:H:Si:O) ((b) spectra extracted from wear track generated while sliding in $\mathrm{H}_{2}$; (c) spectra extracted from wear track generated while sliding in $\mathrm{O}_{2}$ ).

The relative variation in the fraction of carbon rehybridizing from $\mathrm{sp}^{3}-$ to $\mathrm{sp}^{2}$-bonding configuration, computed using Eq. 3 below, is displayed as a function of gas pressure in Figure 4a.

$\Delta f_{s p^{2}}=100 \times \frac{f_{s p^{2}, \text { worn region }}-f_{s p^{2}, \text { unworn region }}}{f_{s p^{2}, \text { unworn region }}}$

Eq. 3

Upon sliding in an oxygen environment, a higher fraction of $\mathrm{C}-\mathrm{C}$ bonds rehybridize from $\mathrm{sp}^{3}$ to $\mathrm{sp}^{2}$ compared to the tests carried out in hydrogen. Additionally, the relative fraction of carbon atoms transitioning from fourfold-coordination to threefold-coordinated decreases with gas pressure.

The higher fraction of carbon atoms rehybridizing from $\mathrm{sp}^{3}$ to $\mathrm{sp}^{2}$ in the experiments performed in oxygen compared to the tests carried out in hydrogen could also explain the larger $\mathrm{C}-\mathrm{H}$ absorption feature at $287.8 \pm 0.2 \mathrm{eV}$ in the difference NEXAFS spectra acquired in the wear tracks generated in oxygen (Figure 3c) relative to the intensity of the same absorption band in the difference NEXAFS spectra collected in the track formed while sliding in hydrogen (Figure 3b). As shown by Michaelson et al. in the case of the dissociative adsorption of molecular deuterium onto poly- and single-crystal diamond surfaces, the dissociation mainly occurs on low hybridized carbon $\left(\mathrm{sp}\right.$ or $\left.\mathrm{sp}^{2}\right)$ states $^{63}$. Thus, the higher fraction of $\mathrm{sp}^{2}$-bonded carbon atoms in the wear track 
present in oxygen environments provides a higher number density of surface sites for the hydrogen dissociative adsorption reaction.

To evaluate changes in carbon hybridization state as a function of depth from the specimen surface, C K-edge spectromicroscopy data were also acquired at different EGB voltages (the higher the EGB voltage, the higher the surface sensitivity or the smaller the information depth ${ }^{41}$, ${ }^{48}$ ). The change in the fraction of $\mathrm{sp}^{2} \mathrm{C}$ atoms with $\mathrm{EGB}$ voltage (Figure $4 \mathrm{~b}$ ) exhibits a positive slope for the majority of the experiments, except for the tests performed at $2000 \mathrm{mbar}$ of hydrogen, i.e., under conditions resulting in a small fraction of carbon atoms rehybridizing from $\mathrm{sp}^{3}$ to $\mathrm{sp}^{2}$ bonding. These positive slopes suggests the presence of a $\mathrm{sp}^{2}$ carbon-rich layer in the near-surface region of tribologically-stressed a-C:H:Si:O, meaning that the tribologically-induced conversion of $\mathrm{sp}^{3}$-bonded C-C bonds occurs more strongly in the outermost surface regions of a-C:H:Si:O (the EGB-dependent escape depth for Auger electrons corresponding to the $\mathrm{C} 1 \mathrm{~s} \rightarrow \sigma^{*}$ transition for $\mathrm{C}$ $\mathrm{H}$ bond was reported to decrease from $3.35 \mathrm{~nm}$ to $1.50 \mathrm{~nm}$ when the EGB voltage is increased from $50 \mathrm{~V}$ to $250 \mathrm{~V})^{48}$. 

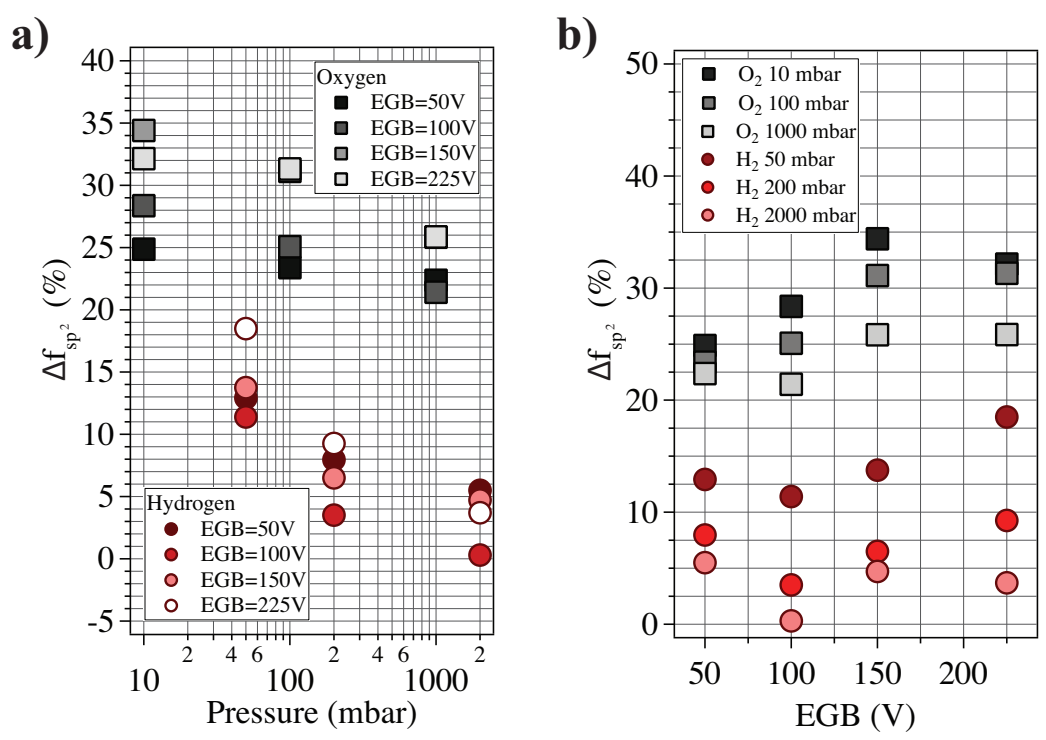

Figure 4. Relative change in the fraction of $\mathrm{sp}^{2}$ carbon as a function of hydrogen and oxygen partial pressure (a) or EGB voltage (b). The surface sensitivity of NEXAFS measurements increases with EGB voltage $\mathrm{e}^{41,48}$.

Since the carbon K-edge NEXAFS spectra of a-C materials are characterized by broad spectral envelopes, which makes it challenging to develop robust physical models including synthetic peaks to be used to fit experimental data, the imaging NEXAFS data reported above were further processed using multivariate analysis (MVA). Figure 5a displays the spectral factors (i.e., $\boldsymbol{S}$ ) estimated from the MCR-ALS utilizing non-negative constraints of the hyperspectral NEXAFS data, as described in section 2.2.4. Figure $5 \mathrm{~b}$ contains the corresponding MCR-ALS refolded spatial factors or intensity maps (i.e., $\boldsymbol{A}$ ). Factor 1 was found to resemble the characteristic carbon K-edge NEXAFS spectrum of as-received a-C:H:Si:O. Its intensity slightly decreased inside the wear tracks, which can be due to the attenuation of this signal by a chemically- and/or structurallydifferent surface layer formed in the wear track, as further suggested by the increase in intensity of factor 2 and 4 inside the wear tracks. Factor 4 is characterized by a very intense feature at 285.0 
$\mathrm{eV}$, thus corresponding to a material with a much higher fraction of $\mathrm{sp}^{2} \mathrm{C}-\mathrm{C}$ bonds (disordered). The intensity of factor 4 increases inside the wear tracks, especially in those generated under oxygen environments. Additionally, it slightly decreases upon increasing the partial pressure of hydrogen or oxygen. These findings are in agreement with the relative change of the fraction of threefold-coordinated carbon atoms reported in Figure 4a. As for factor 2 and 3, their spectra are very similar. However, factor 3 exhibits a larger slope between $286.5 \mathrm{eV}$ and $288 \mathrm{eV}$, thus indicating that this factor corresponds to a material with a larger concentration of $\mathrm{C}-\mathrm{H}$ bonds (the $\mathrm{C} 1 \mathrm{~s} \rightarrow \sigma^{*}$ transition for $\mathrm{C}-\mathrm{H}$ bonds is typically detected at around $287.5 \mathrm{eV}^{41-42,59}$ ). This interpretation is corroborated by the corresponding maps: while the intensity of factor 2 only increases inside the wear tracks generated in oxygen, the intensity of factor 3 decreases inside the tracks formed in oxygen and low hydrogen gas pressure (50 mbar), while it slightly increases for higher hydrogen partial pressure (200 and 2000 mbar). These findings support the conclusions drawn from the computation of the difference between the spectra extracted from both contact and non-contact regions (Figure $3 b$ and $3 c$ ). 

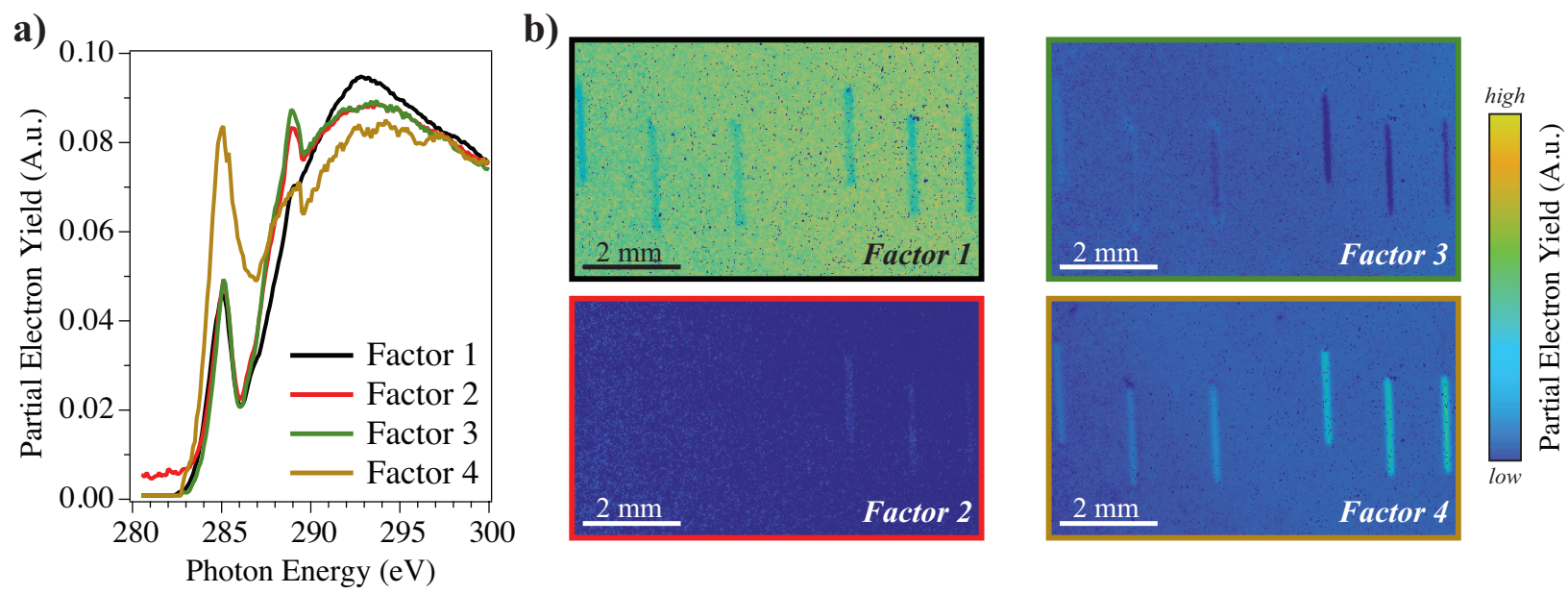

Figure 5. (a) Spectral factors estimated from the MCR-ALS decomposition of hyperspectral carbon K-edge NEXAFS image; and (b) their complementary spatial factor maps. See section 2.2.4 for a description of the methodology for computing these spectral and spatial factors.

In situ (post mortem) XPS measurements were finally performed to quantitatively evaluate changes in surface chemistry of a-C:H:Si:O and determine changes in oxidation state of silicon after tribological tests. The transfer of the specimen from the tribometer chamber to the XPS chamber in vacuum allowed for the characterization of the surface chemistry of wear tracks and out-of-contact regions without any exposure to air and inevitable hydrocarbon contamination. No signals that could be assigned to iron were detected in the survey spectra acquired inside the wear tracks (not shown), indicating that no material was transferred from steel pins to a-C:H:Si:O, in agreement with our previous work ${ }^{37}$. Figure 6a displays the silicon $2 p$ XPS spectra acquired in the contact regions together with the XPS spectrum collected in the non-contact $(n c)$ region. The XPS spectra acquired in the non-contact region indicated the presence of silicon atoms in different oxidation states $(+1$ to +4$)$ in as-deposited a-C:H:Si:O with an oxygen-to-silicon ratio of $0.82 \pm 0.08$. When sliding in hydrogen, a progressive shift of the silicon $2 p$ XPS signal towards 
lower binding energies with gas pressure was detected, which indicate that the fraction of silicon atoms having higher oxidation state decreased with gas pressure ${ }^{64}$ (Figure 6b). This can be due to either the removal of an oxidized surface layer, whose presence was suggested in our previous investigation in which surface-sensitive XPS data were compared with bulk-sensitive RBS/HFS measurements ${ }^{34}$, or to a stress-assisted reduction of silicon. In the case of the sliding tests carried out in oxygen, the silicon 2p XPS spectrum first shifted towards lower binding energies at 10 mbar, which can be again ascribed to the removal of an oxidized surface layer. Increasing the oxygen pressure up to 1000 mbar resulted in a progressive shift of the silicon $2 \mathrm{p}$ peak towards higher binding energies, thus indicating an increase in the fraction of silicon atoms with high oxidation states $^{64}$ (Figure 6b).

The quantification of the composition of the a-C:H:Si:O on the basis of XPS data (Figure $6 \mathrm{c}$ and 6d) indicated that no significant variations occurred when sliding in hydrogen up to 2000 mbar. However, a significant decrease in oxygen concentration was observed at the highest hydrogen pressure (2000 mbar). As for the tests carried out in oxygen, the concentration of silicon and oxygen progressively increase with gas pressure. 

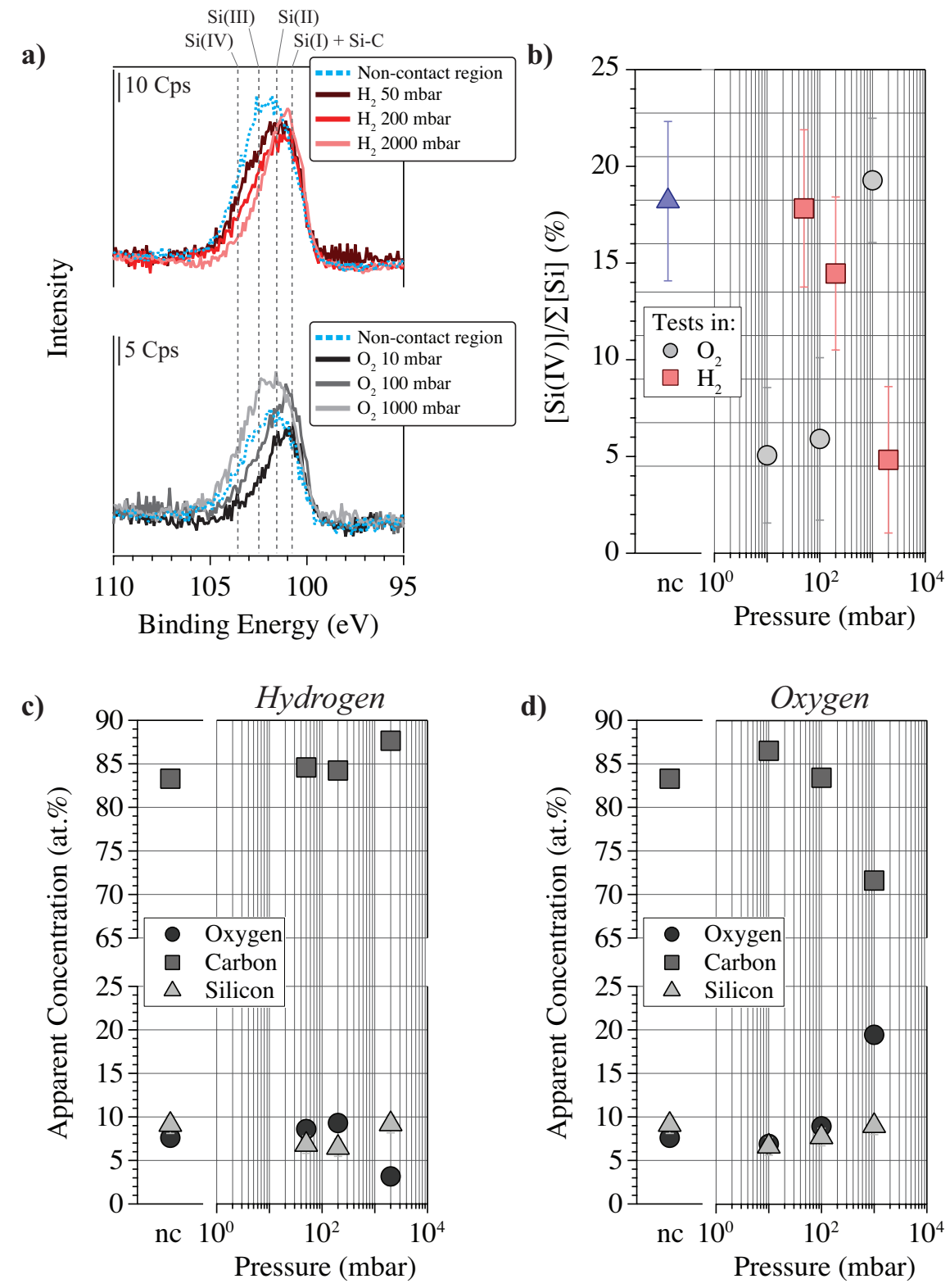

Figure 6. (a) Silicon 2p XPS signals of a-C:H:Si:O obtained from the non-contact region as well as from the wear tracks prepared upon sliding at different hydrogen or oxygen pressure; (b) relative fraction of silicon atoms in oxidation state +4 as a function of gas pressure. The fraction of silicon atoms in oxidation state +4 in the non-contact $(n c)$ region is also reported for comparison; (c) surface composition (calculated on the basis of XPS data) of a-C:H:Si:O as a function of hydrogen pressure; and (d) surface composition (calculated on the basis of XPS data) of a-C:H:Si:O as a 
function of oxygen pressure. The composition of the non-contact $(n c)$ region is also reported for comparison in (c) and (d).

\section{DISCUSSION}

The results of the tribological experiments presented in this study highlighted the dependence of the tribological response of a-C:H:Si:O sliding against steel on the environment, namely the pressure and nature of the gas leaked in the tribometer chamber. More specifically, the coefficient of friction and specific wear rate decrease with the hydrogen partial pressure, whereas they increase with the oxygen partial pressure (between 10 and $10^{3} \mathrm{mbar}$ ). This opposite evolution of the tribological behavior of a-C:H:Si:O with gas pressure for the tests carried out in hydrogen and oxygen clearly suggests a strong dependence of the mechanical response on the surface chemical and structural changes occurring in a-C:H:Si:O upon sliding or at the a-C:H:Si:O/steel sliding interface.

Insights into the tribologically-induced variations in surface chemistry and bonding are gained by ex situ NEXAFS spectromicroscopic measurements performed on a-C:H:Si:O. The spectra extracted from the wear tracks as well as the outcomes of multivariate analysis clearly indicate the tribologically-induced generation of a surface layer containing a higher fraction of disordered $\mathrm{sp}^{2}$ $\mathrm{C}-\mathrm{C}$ bonds together with the presence of a gradient in $\mathrm{sp}^{2}$ carbon concentration as a function of depth from the sample surface (fraction of $\mathrm{sp}^{2}$ carbon increasing in the outermost regions). This finding agrees with our previous study ${ }^{37}$, and with the outcomes of simulations/experiments performed using self-mated diamond contacts, or hydrogenated or hydrogen-free amorphous carbon contacts ${ }^{25-26,43,65-67}$. 
To investigate whether the formation of a surface layer that contains a higher fraction of disordered threefold-coordinated carbon atoms is correlated with the friction behavior of steel sliding against a-C:H:Si:O in hydrogen or oxygen, the variation of the fraction of $\mathrm{sp}^{2}$-hybridized carbon (calculated on the basis of NEXAFS data acquired with $\mathrm{EGB}=100 \mathrm{~V}$. See Figure 4) was plotted as a function of the cumulative energy dissipated during the friction tests (Figure 7). When sliding in hydrogen, increasing the gas pressure reduces the cumulative dissipated energy, and reduces the fraction of carbon atoms that have rehybridized from $\mathrm{sp}^{3}$ - to $\mathrm{sp}^{2}$-bonding. On the contrary, an increase in the cumulative dissipated energy was observed upon increasing the oxygen pressure. However, increasing the oxygen pressure also reduces the fraction of carbon atoms converting from $\mathrm{sp}^{3}$ - to $\mathrm{sp}^{2}$-bonding. This result clearly indicates that the generation of a $\mathrm{sp}^{2}$ bonded carbon-rich layer is not a sufficient condition for achieving low friction independently of the gas environment.

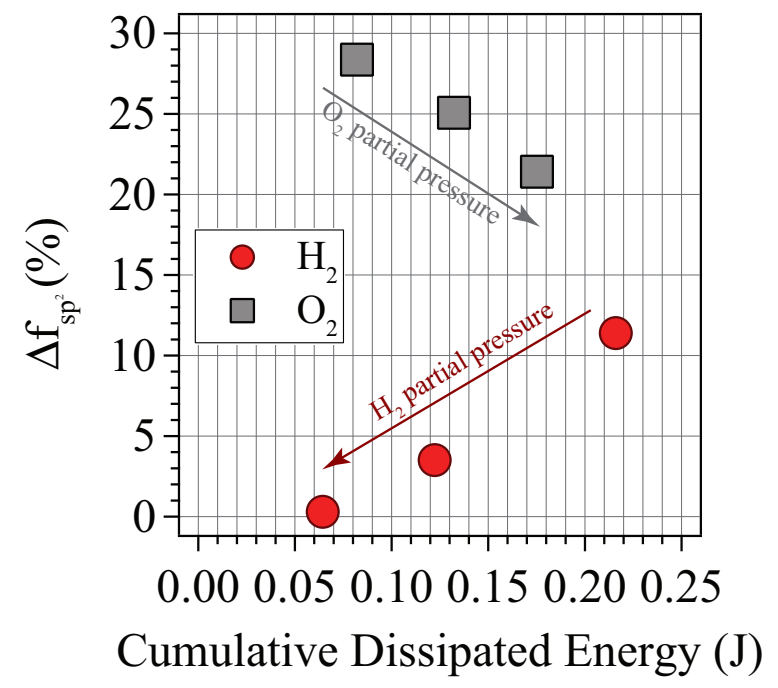

Figure 7. Relative change of the fraction of $\mathrm{sp}^{2}$ carbon (computed from imaging NEXAFS data acquired with EGB voltage equal to $100 \mathrm{~V}$, Figure 4) as a function of the cumulative energy dissipated during sliding experiments (integral under the friction force $v s$. sliding distance curve). 
Based on these findings and on the outcomes of the surface-analytical investigations of a$\mathrm{C}: \mathrm{H}: \mathrm{Si}: \mathrm{O}$ after sliding experiments, the following model is proposed for explaining the tribological behavior of steel/a-C:H:Si:O contacts (Figure 8 ) where gas pressures are sufficiently high to avoid the adhesive transfer of steel to the a-C:H:Si:O surface, i.e., in the low friction regime. While sliding, nanoscale asperities on the surface of a-C:H:Si:O film and steel pin collide and contacts are formed (Stage 1 and 2). As shown by Kunze et al. ${ }^{26}$ and Le Huu et al. ${ }^{65}$, a stress-induced rehybridization of carbon atoms from $\mathrm{sp}^{3}$ - to $\mathrm{sp}^{2}$-bonding occurs during deformation of these asperities. The resulting highly strained $\mathrm{sp}^{2} \mathrm{C}-\mathrm{C}$ bonds at sliding interfaces ${ }^{26}$ are prone to react with molecules in the environment ${ }^{68}$ (Stage 3 ), such as highly reactive gases (e.g., $\mathrm{H}_{2}$ or $\mathrm{O}_{2}$ ), and, thus, easily break, as proposed in our previous work ${ }^{37}$. The reaction between strained $\mathrm{sp}^{2} \mathrm{C}-\mathrm{C}$ bonds and gas species, whose rate is dependent on the gas and its partial pressure, not only leads to the generation of a transfer layer on the steel pin, but also leaves a fully-passivated surface with a higher fraction of $\mathrm{sp}^{2}$-hybridized carbon atoms compared to the one in the bulk of a-C:H:Si:O.

When sliding in hydrogen, hydrogen molecules react with strained $\mathrm{C}-\mathrm{C} \mathrm{sp}{ }^{2}$ bonds to create a hydrogenated amorphous carbon layer with low shear strength (Stage 4), consistent with the study of Scharf et al. ${ }^{28}$. The surface passivation of a-C:H:Si:O with hydrogen atoms also precludes covalent bonds to be formed across the sliding interface ${ }^{1,} 16,69$. Increasing the hydrogen gas pressure increases the rate of the reaction occurring at the sliding interface, which results in a fractional increase of $\mathrm{C}-\mathrm{H}$ bonds in the near-surface region, thus decreasing the shear strength of the material at the sliding interface. This is proposed to reduce friction with gas pressure.

Upon sliding in oxygen, the strained $\mathrm{C}-\mathrm{C} \mathrm{sp}^{2}$ bonds react with oxygen molecules, whose dissociative adsorption results in the generation of surface carbonyl/ether groups. In our previous 
work ${ }^{37}$, the formation of an interfacial material with a high degree of saturation was proposed to lead to low friction for steel/a-C:H:Si:O sliding contacts at an oxygen pressure of 10 mbar. This hypothesis is supported by published literature that highlighted the key role of ether/keto groups in inhibiting the formation of strong $\mathrm{C}-\mathrm{C}$ covalent bonds across the sliding interface between diamond surfaces, thus aiding in friction reduction ${ }^{70}$. While the formation of additional $\mathrm{C}=\mathrm{O} / \mathrm{C}-\mathrm{O}$ groups could increase the shear strength of the interfacial material and/or contribute to the formation of ether linkages across the interface, the absolute intensity of the signal assigned to $\mathrm{C}=\mathrm{O} / \mathrm{C}-\mathrm{O}$ bonds in difference NEXAFS spectra (Figure $3 \mathrm{c}$ ) decreased with gas pressure, thus not explaining the progressive increase in friction of a-C:H:Si:O with oxygen pressure. However, in situ (post mortem) X-ray photoelectron spectroscopy analyses provide insights into the gaspressure dependent friction response for a-C:H:Si:O: the oxygen content in the outermost region of a-C:H:Si:O increased with the oxygen partial pressure together with a continuous increase in the fraction of silicon atoms that are in high oxidation state. These changes are proposed to lead to the formation of an interfacial material with a progressively higher shear strength, thus increasing friction with gas pressure.

The slight changes in surface composition of the wear tracks generated on a-C:H:Si:O with gas pressure observed in the present study is consistent with the results of ToF-SIMS analyses carried out by Scharf et al. on tribotracks formed by a silicon nitride pin sliding on a-C:H:Si: ${ }^{28}$, which indicated small variations in intensity for the characteristic fragments of a-C:H:Si:O (e.g., $\mathrm{O}^{-}, \mathrm{SiO}_{2}{ }^{-}$ , $\left.\mathrm{Si}^{-}, \mathrm{C}_{1}^{-}\right)$inside the wear tracks compared to the out-of-contact region independently of the gas environment (humid air, dry air, or dry nitrogen). The ToF-SIMS characterization of silicon nitride pins provided evidence for the strong influence of the environment on the chemistry of tribofilms formed on countersurfaces: in dry nitrogen, tribofilms were rich in hydrogenated carbon- 
containing species, while in humid conditions they contained significant amounts of silicon and silicon oxide fragments. These variations in tribofilm chemistry formed on silicon nitride pins was correlated with the interfacial shear strength $(9 \mathrm{MPa}$ in dry nitrogen and $78 \mathrm{MPa}$ in humid conditions).

While further studies are required to quantitatively evaluate the chemistry and structure of tribofilms formed on the steel pins during sliding on a-C:H:Si:O, the present work demonstrates, for the first time, that a-C:H:Si:O undergoes environmentally-dependent changes in carbon bonding configuration and silicon oxidation state, which affect the shear strength of the interfacial material.

a)

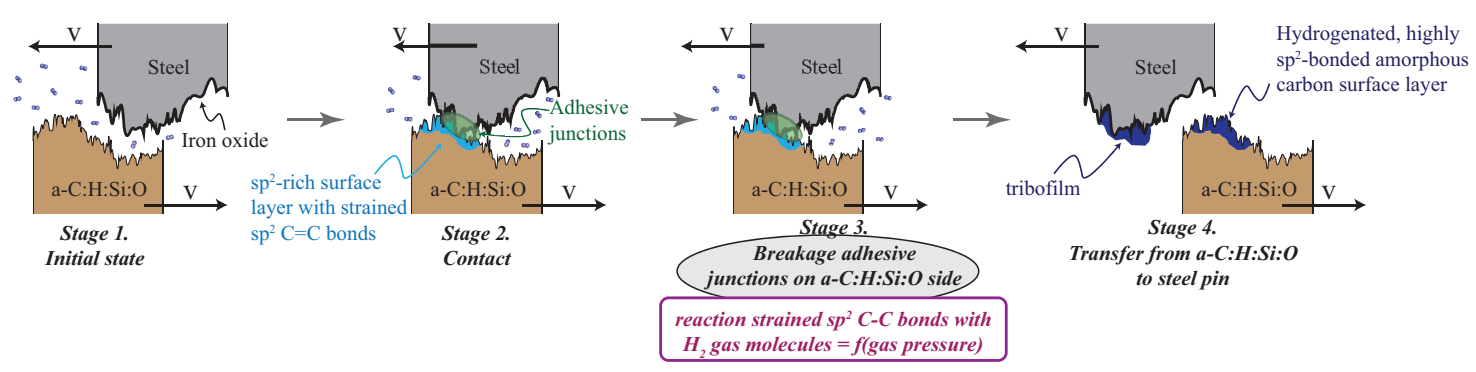

b)

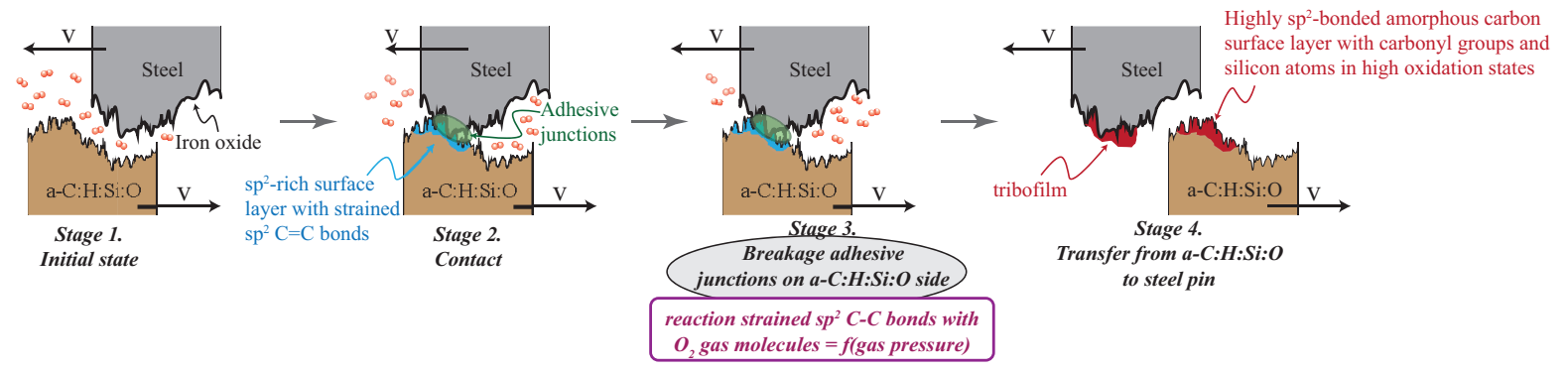

Figure 8. Model proposed to describe the friction response of a steel vs. a-C:H:Si:O tribosystem sliding in hydrogen (a) or oxygen (b) atmosphere. 


\section{CONCLUSIONS}

The tribological response of a steel pin sliding against a-C:H:Si:O was evaluated as a function of the partial pressure of oxygen and hydrogen in vacuum in the low friction/low wear regime, where sufficient gas pressure prevents steel adhering to the a-C:H:Si:O, with the velocity accommodation mode being interfacial sliding between the wear track and the carbonaceous tribofilm formed on the countersurface. While the coefficient of friction and wear rate in this regime increase with the oxygen partial pressure, they decrease with the hydrogen partial pressure. The post-mortem analysis of a-C:H:Si:O flats by in situ XPS and ex situ NEXAFS spectromicroscopy together with the use, for the first time, of multivariate analysis (MVA) methods to process complex surface-analytical spectroscopic data, allowed for the identification of the surface chemical and structural changes occurring on a-C:H:Si:O upon sliding in different environmental conditions. The analytical results indicated the stress-induced $\mathrm{sp}^{3}$-to-sp ${ }^{2}$ rehybridization of $\mathrm{C}-\mathrm{C}$ bonds near the surface. The strained $\mathrm{sp}^{2} \mathrm{C}-\mathrm{C}$ bonds generated at sliding interfaces are prone to reacting with gas molecules. In the case of the sliding tests carried out in hydrogen, this reaction results in the formation of a hydrogenated amorphous carbon interfacial material on the flat, in which the fraction of $\mathrm{C}-\mathrm{H}$ bonds increases with gas pressure. The progressive decrease of the shear strength of the interfacial material is proposed to be the reason behind the decrease of friction with hydrogen pressure. As for the experiments in oxygen environments, the reaction of strained $\mathrm{sp}^{2} \mathrm{C}-\mathrm{C}$ bonds at the sliding interface with oxygen molecules leads to a progressive increase in oxygen content in outermost regions of a-C:H:Si:O. This, together with a higher percentage of silicon atoms with high oxidation states, increases the shear strength of the interfacial material, which is proposed to cause an increase in friction with oxygen partial pressure. 


\section{AUTHOR INFORMATION}

\section{Corresponding Author}

*Email: carpick@,seas.upenn.edu

\section{Notes}

The authors declare no competing financial interests.

\section{SUPPORTING INFORMATION}

Typical friction force vs. slider position during one reciprocating cycle.

\section{ACKNOWLEDGMENTS}

This material is based upon work supported by the Agence Nationale de la Recherche under grant No. ANR-11-NS09-01 and by the National Science Foundation under Grant No. DMR1107642 and through the Materials World Network program. F.M. acknowledges support from the Welch Foundation under Grant F-2002-20190330 and the Marie Curie International Outgoing Fellowship under contract no. PIOF-GA-2012-328776. K.K. acknowledges Région Rhône-Alpes and Université de Lyon for their support through CMIRA and PALSE program respectively. F.M., J.B.M., and R.W.C. acknowledge support from the Advanced Storage Technology Consortium (grant 2011-012). J.B.M. acknowledges support of a Graduate Research Supplement for Veterans from the Directorate for Mathematical and Physical Sciences of the National Science Foundation. The authors would like to thank Dr. D.A. Fischer and Dr. C. Jaye for their help with NEXAFS measurements at the National Synchrotron Light Source. Use of the National Synchrotron Light Source, Brookhaven National Laboratory, was supported by the US Department of Energy, Office 
of Science and Office of Basic Energy Sciences, under Contract No. DE-AC02-98CH10886. We acknowledge Th. Le Mogne and M. Guibert for their support with the environment-controlled tribometer. Sandia National Laboratories is a multimission laboratory managed and operated by National Technology \& Engineering Solutions of Sandia, LLC, a wholly owned subsidiary of Honeywell International Inc., for the U.S. Department of Energy's National Nuclear Security Administration under contract DE-NA0003525. This paper describes objective technical results and analysis. Any subjective views or opinions that might be expressed in the paper do not necessarily represent the views of the U.S. Department of Energy or the United States Government. 


\section{REFERENCES}

1. Donnet, C.; Erdemir, A., Tribology of Diamond-Like Carbon Films. Springer: New York, 2008; p. 664.

2. Fontaine, J., Towards the use of diamond-like carbon solid lubricant coatings in vacuum and space environments. Proceedings of the Institution of Mechanical Engineers, Part J: Journal of Engineering Tribology 2008, 222 (8), 1015-1029.

3. Holmberg, K.; Andersson, P.; Erdemir, A., Global energy consumption due to friction in passenger cars. Tribology International 2012, 47, 221-234.

4. Heaney, P. J.; Sumant, A. V.; Torres, C. D.; Carpick, R. W.; Pfefferkorn, F. E., Diamond coatings for micro end mills: Enabling the dry machining of aluminum at the micro-scale. Diamond and Related Materials 2008, 17 (3), 223-233.

5. Auciello, O.; Birrell, J.; Carlisle, J. A.; Gerbi, J. E.; Xiao, X.; Peng, B.; Espinosa, H. D., Materials science and fabrication processes for a new MEMS technology based on ultrananocrystalline diamond thin films. Journal of Physics: Condensed Matter 2004, 16 (16), R539-R552.

6. Liu, J.; Grierson, D. S.; Moldovan, N.; Notbohm, J.; Li, S.; Jaroenapibal, P.; O'Connor, S. D.; Sumant, A. V.; Neelakantan, N.; Carlisle, J. A.; Turner, K. T.; Carpick, R. W., Preventing Nanoscale Wear of Atomic Force Microscopy Tips Through the Use of Monolithic Ultrananocrystalline Diamond Probes. Small 2010, 6 (10), 1140-1149.

7. Ferrari, A. C., Diamond-like carbon for magnetic storage disks. Surface and Coatings Technology 2004, 180-181, 190-206. 
8. Erdemir, A.; Bindal, C.; Pagan, J.; Wilbur, P., Characterization of transfer layers on steel surfaces sliding against diamond-like hydrocarbon films in dry nitrogen. Surface and Coatings Technology 1995, 76-77 (Part 2), 559-563.

9. Liu, Y.; Erdemir, A.; Meletis, E. I., An investigation of the relationship between graphitization and frictional behavior of DLC coatings. Surface and Coatings Technology 1996, 86-87 (Part 2), 564-568.

10. Li, H.; Xu, T.; Wang, C.; Chen, J.; Zhou, H.; Liu, H., Tribochemical effects on the friction and wear behaviors of diamond-like carbon film under high relative humidity condition. Tribology Letters 2005, 19 (3), 231-238.

11. Kim, H. I.; Lince, J. R.; Eryilmaz, O. L.; Erdemir, A., Environmental effects on the friction of hydrogenated DLC films. Tribology Letters 2006, 21 (1), 51-56.

12. Mangolini, F.; Rose, F.; Hilbert, J.; Carpick, R. W., Thermally induced evolution of hydrogenated amorphous carbon. Applied Physics Letters 2013, 103 (16), 161605.

13. Robertson, J., Diamond-like amorphous carbon. Materials Science and Engineering: $R$ : Reports 2002, 37 (4-6), 129-281.

14. Singer, I. L.; Dvorak, S. D.; Wahl, K. J.; Scharf, T. W., Role of third bodies in friction and wear of protective coatings. Journal of Vacuum Science \& Technology A: Vacuum, Surfaces, and Films 2003, 21 (5), S232-S240.

15. Pastewka, L.; Moser, S.; Moseler, M., Atomistic Insights into the Running-in, Lubrication, and Failure of Hydrogenated Diamond-Like Carbon Coatings. Tribology Letters 2010, 39 (1), 4961. 
16. Fontaine, J.; Belin, M.; Le Mogne, T.; Grill, A., How to restore superlow friction of DLC: the healing effect of hydrogen gas. Tribology International 2004, 37 (11-12), 869-877.

17. Olsen, J. E.; Fischer, T. E.; Gallois, B., In situ analysis of the tribochemical reactions of diamond-like carbon by internal reflection spectroscopy. Wear 1996, 200 (1-2), 233-237.

18. Andersson, J.; Erck, R. A.; Erdemir, A., Frictional behavior of diamondlike carbon films in vacuum and under varying water vapor pressure. Surface and Coatings Technology 2003, 163 $164,535-540$.

19. Harrison, J. A.; Brenner, D. W., Simulated Tribochemistry: An Atomic-Scale View of the Wear of Diamond. Journal of the American Chemical Society 1994, 116 (23), 10399-10402.

20. Moras, G.; Pastewka, L.; Gumbsch, P.; Moseler, M., Formation and Oxidation of Linear Carbon Chains and Their Role in the Wear of Carbon Materials. Tribology Letters 2011, 44 (3), $355-365$.

21. Qi, Y.; Konca, E.; Alpas, A. T., Atmospheric effects on the adhesion and friction between non-hydrogenated diamond-like carbon (DLC) coating and aluminum - A first principles investigation. Surface Science 2006, 600 (15), 2955-2965.

22. Zilibotti, G.; Righi, M. C.; Ferrario, M., Ab initio study on the surface chemistry and nanotribological properties of passivated diamond surfaces. Physical Review B 2009, 79 (7), 075420 .

23. van den Oetelaar, R. J. A.; Flipse, C. F. J., Atomic-scale friction on diamond(111) studied by ultra-high vacuum atomic force microscopy. Surface Science 1997, 384 (1-3), L828-L835. 
24. Erdemir, A.; Fenske, G. R.; Krauss, A. R.; Gruen, D. M.; McCauley, T.; Csencsits, R. T., Tribological properties of nanocrystalline diamond films. Surface and Coatings Technology 1999, $120-121,565-572$.

25. Konicek, A. R.; Grierson, D. S.; Gilbert, P. U. P. A.; Sawyer, W. G.; Sumant, A. V.; Carpick, R. W., Origin of Ultralow Friction and Wear in Ultrananocrystalline Diamond. Physical Review Letters 2008, 100 (23), 235502.

26. Kunze, T.; Posselt, M.; Gemming, S.; Seifert, G.; Konicek, A. R.; Carpick, R. W.; Pastewka, L.; Moseler, M., Wear, Plasticity, and Rehybridization in Tetrahedral Amorphous Carbon. Tribology Letters 2013, 53 (1), 119-126.

27. Sánchez-López, J. C.; Fernández, A., Doping and Alloying Effects on DLC Coatings. In Tribology of Diamond-Like Carbon Films: Fundamentals and Applications., Donnet, C.; Erdemir, A., Eds. Springer: 2008, pp 311-338.

28. Scharf, T. W.; Ohlhausen, J. A.; Tallant, D. R.; Prasad, S. V., Mechanisms of friction in diamondlike nanocomposite coatings. Journal of Applied Physics 2007, 101 (6), 063521.

29. Yang, W. J.; Choa, Y.-H.; Sekino, T.; Shim, K. B.; Niihara, K.; Auh, K. H., Thermal stability evaluation of diamond-like nanocomposite coatings. Thin Solid Films 2003, 434 (1-2), 49-54.

30. Jongwannasiri, C.; Li, X.; Watanabe, S., Improvement of Thermal Stability and Tribological Performance of Diamond-Like Carbon Composite Thin Films. Materials Science and Applications 2013, 4, 630-636. 
31. Neerinck, D.; Persoone, P.; Sercu, M.; Goel, A.; Kester, D.; Bray, D., Diamond-like nanocomposite coatings (a-C:H/a-Si:O) for tribological applications. Diamond and Related Materials 1998, 7 (2-5), 468-471.

32. Mangolini, F.; Hilbert, J.; McClimon, J. B.; Lukes, J. R.; Carpick, R. W., Thermally Induced Structural Evolution of Silicon- and Oxygen-Containing Hydrogenated Amorphous Carbon: A Combined Spectroscopic and Molecular Dynamics Simulation Investigation. Langmuir 2018, 34 (9), 2989-2995.

33. Hilbert, J.; Mangolini, F.; McClimon, J. B.; Lukes, J. R.; Carpick, R. W., Si doping enhances the thermal stability of diamond-like carbon through reductions in carbon-carbon bond length disorder. Carbon 2018, 131, 72-78.

34. Mangolini, F.; Krick, B. A.; Jacobs, T. D. B.; Khanal, S. R.; Streller, F.; McClimon, J. B.; Hilbert, J.; Prasad, S. V.; Scharf, T. W.; Ohlhausen, J. A.; Lukes, J. R.; Sawyer, W. G.; Carpick, R. W., Effect of silicon and oxygen dopants on the stability of hydrogenated amorphous carbon under harsh environmental conditions. Carbon 2018, 130, 127-136.

35. Scharf, T. W.; Singer, I. L., Third Bodies and Tribochemistry of DLC Coatings. In Tribology of Diamond-Like Carbon Films: Fundamentals and Applications, Donnet, C.; Erdemir, A., Eds. Springer: New York, 2008, pp 201-236.

36. Scharf, T. W.; Singer, I. L., Role of Third Bodies in Friction Behavior of Diamond-like Nanocomposite Coatings Studied by In Situ Tribometry. Tribology Transactions 2002, 45 (3), 363-371. 
37. Koshigan, K. D.; Mangolini, F.; McClimon, J. B.; Vacher, B.; Bec, S.; Carpick, R. W.; Fontaine, J., Understanding the hydrogen and oxygen gas pressure dependence of the tribological properties of silicon oxide-doped hydrogenated amorphous carbon coatings. Carbon 2015, 93, 851-860.

38. Gilmore, R.; Hauert, R., Control of the tribological moisture sensitivity of diamond-like carbon films by alloying with F, Ti or Si. Thin Solid Films 2001, 398-399, 199-204.

39. Peng, J.; Sergiienko, A.; Mangolini, F.; Stallworth, P. E.; Greenbaum, S.; Carpick, R. W., Solid state magnetic resonance investigation of the thermally-induced structural evolution of silicon oxide-doped hydrogenated amorphous carbon. Carbon 2016, 105, 163-175.

40. Mangolini, F.; McClimon, J. B.; Carpick, R. W., Quantitative Evaluation of the Carbon Hybridization State by Near Edge X-ray Absorption Fine Structure Spectroscopy. Anal Chem 2016, 88 (5), 2817-24.

41. Mangolini, F.; McClimon, J. B.; Rose, F.; Carpick, R. W., Accounting for nanometer-thick adventitious carbon contamination in X-ray absorption spectra of carbon-based materials. Anal Chem 2014, 86 (24), 12258-12265.

42. Sumant, A. V.; Gilbert, P. U. P. A.; Grierson, D. S.; Konicek, A. R.; Abrecht, M.; Butler, J. E.; Feygelson, T.; Rotter, S. S.; Carpick, R. W., Surface composition, bonding, and morphology in the nucleation and growth of ultra-thin, high quality nanocrystalline diamond films. Diamond and Related Materials 2007, 16 (4-7), 718-724.

43. Konicek, A. R.; Grierson, D. S.; Sumant, A. V.; Friedmann, T. A.; Sullivan, J. P.; Gilbert, P. U. P. A.; Sawyer, W. G.; Carpick, R. W., Influence of surface passivation on the friction and 
wear behavior of ultrananocrystalline diamond and tetrahedral amorphous carbon thin films. Physical Review B 2012, 85 (15), 155448.

44. Miyoshi, K.; Iwaki, M.; Gotoh, K.; Obara, K., Friction and Wear Properties of Selected Solid Lubricating Films. NASA/TM-1999-209088, 1999.

45. Pepper, S. V.; Ebihara, B.T.; Kingsbury, E. A Rolling Element Tribometer for the Study of Liquid Lubricants in Vacuum. NASA/TP-3629; 1996.

46. Konicek, A.; Jaye, C.; Hamilton, M.; Sawyer, W.; Fischer, D.; Carpick, R., Near-Edge Xray Absorption Fine Structure Imaging of Spherical and Flat Counterfaces of Ultrananocrystalline Diamond Tribological Contacts: A Correlation of Surface Chemistry and Friction. Tribology Letters 2011, 44 (1), 99-106.

47. Mangolini, F.; McClimon, J. B., Near Edge X-Ray Absorption Fine Structure Spectroscopy: A Powerful Tool for Investigating the Surface Structure and Chemistry of Solid Lubricants. In Advanced Analytical Methods in Tribology, Dienwiebel, M.; De Barros Bouchet, M. I., Eds. Springer: 2018; Chapter Chapter 3, pp 63-106.

48. Genzer, J.; Kramer, E. J.; Fischer, D. A., Accounting for Auger yield energy loss for improved determination of molecular orientation using soft X-ray absorption spectroscopy. Journal of Applied Physics 2002, 92 (12), 7070-7079.

49. Mangolini, F.; Rossi, A.; Spencer, N. D., Chemical Reactivity of Triphenyl Phosphorothionate (TPPT) with Iron: An ATR/FT-IR and XPS Investigation. The Journal of Physical Chemistry C 2011, 115 (4), 1339-1354. 
50. Powell, C. J., The Physical Basis for Quantitative Surface Analysis by Auger Electron Spectroscopy and X-ray Photoelectron Spectroscopy. In Quantitative Surface Analysis of Materials, McIntyre, N. S., Ed. American Society for Testing and Materials: 1978, pp 5-30.

51. Tanuma, S., Electron Attenuation Lengths. In Surface Analysis by Auger and X-Ray Photoelectron Spectroscopy, Briggs, D.; Grant, J. T., Eds. IM Publications: Chichester (UK), 2003; Chapter 11, pp 259-294.

52. Rodriguez, M. A.; Van Benthem, M. H.; Ingersoll, D.; Vogel, S. C.; Reiche, H. M., In situ analysis of $\mathrm{LiFePO}_{4}$ batteries: Signal extraction by multivariate analysis. Powder Diffraction 2012, $25(2), 143-148$.

53. Keenan, M. R., Multivariate Analysis of Spectral Images Composed of Count Data. In Techniques and Applications of Hyperspectral Image Analysis, 2007, pp 89-126.

54. Rummel, R. J., Applied Factor Analysis. Northwestern University Press: Evanston, IL, 1988; p 617.

55. Harman, H. H., Modern Factor Analysis. University of Chicago Press: Chicago, IL, 1976; p 487.

56. Keenan, M. R.; Kotula, P. G., Accounting for Poisson noise in the multivariate analysis of ToF-SIMS spectrum images. Surface and Interface Analysis 2004, 36 (3), 203-212.

57. Rodriguez, M. A.; Keenan, M. R.; Nagasubramanian, G., In situ X-ray diffraction analysis of $\left(\mathrm{CF}_{\mathrm{x}}\right)_{\mathrm{n}}$ batteries: signal extraction by multivariate analysis. Journal of Applied Crystallography 2007, 40 (6), 1097-1104. 
58. Van Benthem, M. H.; Keenan, M. R., Tucker1 model algorithms for fast solutions to large PARAFAC problems. Journal of Chemometrics 2008, 22 (5), 345-354.

59. Stöhr, J., NEXAFS Spectroscopy. Springer-Verlag Berlin Heidelberg: 1992; p 404.

60. Comelli, G.; Stöhr, J.; Robinson, C. J.; Jark, W., Structural studies of argon-sputtered amorphous carbon films by means of extended x-ray-absorption fine structure. Physical Review B 1988, $38(11), 7511-7519$.

61. Ishii, I.; Hitchcook, A. P., The oscillator strengths for $\mathrm{C} 1 \mathrm{~s}$ and $\mathrm{O} 1 \mathrm{~s}$ excitation of some saturated and unsaturated organic alcohols, acids and esters. Journal of Electron Spectroscopy and Related Phenomena 1988, 46 (1), 55-84.

62. Wada, A.; Ogaki, T.; Niibe, M.; Tagawa, M.; Saitoh, H.; Kanda, K.; Ito, H., Local structural analysis of $\mathrm{a}-\mathrm{SiC}_{\mathrm{x}}: \mathrm{H}$ films formed by decomposition of tetramethylsilane in microwave discharge flow of Ar. Diamond and Related Materials 2011, 20 (3), 364-367.

63. Michaelson, S.; Chandran, M.; Zalkind, S.; Shamir, N.; Akhvlediani, R.; Hoffman, A., Dissociative adsorption of molecular deuterium and thermal stability onto hydrogenated, bare and ion beam damaged poly- and single crystalline diamond surfaces. Surface Science 2015, 642, 1621.

64. Dreiner, S.; Schürmann, M.; Westphal, C., Investigation of the $\mathrm{SiO}_{2} / \mathrm{Si}\left(\begin{array}{lll}1 & 0 & 0\end{array}\right)$ interface structure by means of angle-scanned photoelectron spectroscopy and diffraction. Journal of Electron Spectroscopy and Related Phenomena 2004, 137-140, 79-84. 
65. Le Huu, T.; Zaidi, H.; Paulmier, D.; Voumard, P., Transformation of $\mathrm{sp}^{3}$ to $\mathrm{sp}^{2}$ sites of diamond like carbon coatings during friction in vacuum and under water vapour environment. Thin Solid Films 1996, 290-291 (0), 126-130.

66. Li, L.; Song, W.; Ovcharenko, A.; Xu, M.; Zhang, G., Effects of atomic structure on the frictional properties of amorphous carbon coatings. Surface and Coatings Technology 2015, 263, 8-14.

67. Liu, Q.; Li, L.; Zhang, H.; Huang, Q.; Zhang, G.; Hou, Z., Investigation on the Graphitization Process of Amorphous Carbon for HAMR by Molecular Dynamics Simulation. IEEE Transactions on Magnetics 2017, 53 (3), 1-7.

68. Matta, C.; De Barros Bouchet, M. I.; Le-Mogne, T.; Vachet, B.; Martin, J. M.; Sagawa, T., Tribochemistry of tetrahedral hydrogen-free amorphous carbon coatings in the presence of OHcontaining lubricants. Lubrication Science 2008, 20 (2), 137-149.

69. Gao, G. T.; Mikulski, P. T.; Harrison, J. A., Molecular-Scale Tribology of Amorphous Carbon Coatings: Effects of Film Thickness, Adhesion, and Long-Range Interactions. Journal of the American Chemical Society 2002, 124 (24), 7202-7209.

70. Kuwahara, T.; Moras, G.; Moseler, M., Role of oxygen functional groups in the friction of water-lubricated low-index diamond surfaces. Physical Review Materials 2018, 2 (7), 073606. 


\section{TOC GRAPHICS}
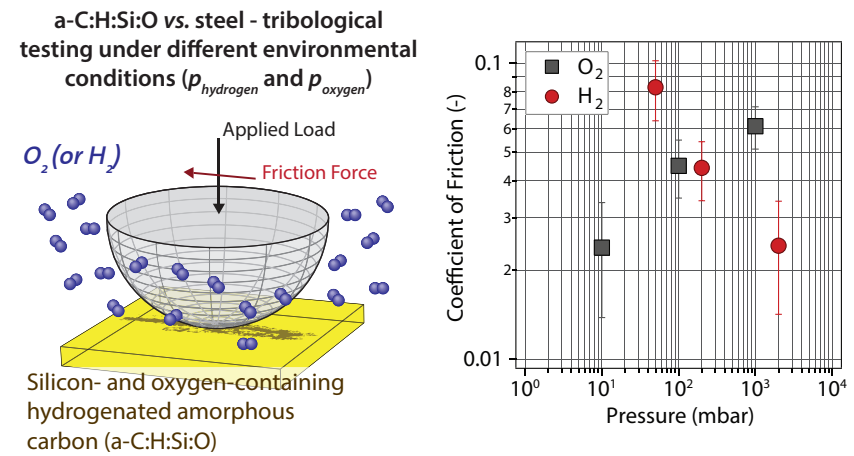